\title{
Psychosocial Determinants of Cannabis Dependence: A Systematic Review of the Literature
}

\author{
Samantha Schlossarek ${ }^{a}$ Jürgen Kempkensteffen ${ }^{b}$ Jens Reimer ${ }^{a}$ \\ Uwe Verthein ${ }^{a}$ \\ a Department of Psychiatry, Centre for Interdisciplinary Addiction Research of Hamburg University, University \\ Medical Centre Hamburg-Eppendorf, and b Department of Clinical Psychology and Psychotherapy, University of \\ Hamburg, Institute for Psychology, Hamburg, Germany
}

\section{Key Words}

Cannabis dependence - Cannabis use - Risk factors .

Psychosocial determinants

\begin{abstract}
Background: Many studies have examined factors associated with the first onset of cannabis use and abuse. Currently, there is relatively little research regarding conditions under which cannabis dependence is more likely to emerge. Although previous studies have examined different potential determinants of cannabis dependence, to our knowledge, a systematic review is lacking. Aims: The study aims to identify recent findings regarding psychosocial determinants of cannabis dependence and to summarize them systematically. Methods: A literature search in 4 databases - Embase, Medline, PsycINFO and PSYNDEX - was conducted. Searches were limited to publications between 2000 and April 2014, English and German as languages and humans as study subjects. Results: Our search detected a total of 10,568 studies. Twenty-six studies finally met inclusion criteria. Consumption patterns such as a regular cannabis use independent of social context and an early onset of use (11-15 years) were correlates of cannabis dependence. Moreover, early reactions to cannabis use and coping-oriented use motives ex-
\end{abstract}

plained additional variance. Stress factors and critical life events such as parental separation and early parental death as well as mental and social conflicts have also been linked with development of cannabis dependence. Additionally, comorbid mental disorders correlated with cannabis dependence. Conclusion: Numerous factors were shown to have an impact on transition to cannabis dependence. In particular, a wide range of mental disorders has been linked to an elevated risk of becoming dependent. The development of a dependence syndrome seems to be associated with diverse processes, in which social, biological and intra-individual factors interact in a complex manner. Nevertheless, the link between cannabis dependence and predisposing factors could not be resolved convincingly by most studies due to methodological weaknesses regarding dependence criteria.

(c) 2015 S. Karger AG, Basel

\section{Introduction}

Cannabis use, its risk potential and its predominantly current illegal status in most western countries are currently debated issues [1-4]. As a result, facts and contentious assumptions - so-called 'cannabis myths' - sur-

\section{KARGER}

E-Mail karger@karger.com www.karger.com/ear
(C) 2015 S. Karger AG, Basel

$1022-6877 / 15 / 0223-0131 \$ 39.50 / 0$
Uwe Verthein

Centre for Interdisciplinary Addiction Research of Hamburg University Department of Psychiatry, University Medical Centre Hamburg Eppendorf Martinistrasse 52, DE-20246 Hamburg (Germany)

E-Mail u.verthein@uke.de 
round the substance. These myths emerge without clear empirical evidence, for example, the theory of cannabis as a gateway drug, as well as the causation of apathy and loss in motivation ('amotivational syndrome') $[5,6]$. Furthermore, risks of cannabis use are consistently weighed up against its benefits $[7,8]$. On one hand, some studies reported that cannabis use causes psychotic symptoms and that the use of cannabis by itself, that is, without any cofactors, leads directly to development of dependence or causes the use of other drugs (gateway hypothesis). On the other hand, cannabis seems to have a therapeutic potential, for example alleviating pain or nausea in persons with multiple sclerosis and cancer [7,9-11]. Accordingly, the call for legalization arises repeatedly from time to time, in response to prohibitionist policies toward cannabis use in many societies [4]. Some countries have even relaxed legal constraints on cannabis use and its possession, like the recent cases in Washington and Colorado $[12,13]$. In the last years, the concentration of delta-9-tetrahydrocannabinol as the primary psychoactive compound has increased and therefore the effect on users may be stronger $[2,14]$. This fact may also have contributed to a controversy surrounding its harmfulness and its increased potential for addiction $[7,15]$.

Moreover, cannabis is the world's most prevalent illicit drug and the third most commonly used psychoactive substance, after alcohol and tobacco $[2,7,8,16]$. At the same time, there seems to be a decrease in global cannabis use. Although having considerably higher rates of past-year users than the global average, Europe seems to exhibit stable or even declining rates of cannabis use, particularly in younger age groups $[2,17]$. Estimates from 2014 EMCDDA indicated that $21.7 \%$ of European adults have reported lifetime use, and 5.3\% have used cannabis in the past year [2]. In addition, only a minority were (almost) daily users, namely under $1 \%$ of European adults; over two-thirds of these (almost) daily users were between 15 and 34 years of age. In this age group, over threequarters were men [2].

Estimates of the 12-month prevalence concerning cannabis dependence vary widely from 0.5 to $42.0 \%$ [18-21]. This variation in prevalence can be explained by different samples (representative sample [18, 21], cannabis users [19] and students [20]), and sampling techniques (probability sampling [18, 21], snowball sampling [19] and convenience sampling [20]). In the United States, $1.7 \%$ of the total population fulfilled criteria for cannabis dependence or abuse in the past year. This prevalence was similar to the years before, ranging between 1.6 and $1.8 \%$ [16]. If one compares rates of cannabis use with those of dependence, for most people, use of cannabis is controlled and limited to a short period of time and does not result in abuse or dependence $[22,23]$.

Cannabis dependence is characterized by compulsive use, impaired control and continued use despite physical and psychological problems caused or exacerbated by such drug use [24, 25]. For a diagnosis, one needs to meet a minimum of 3 of 6 criteria of the ICD-10 [26] or 3 of 7 criteria of the DSM-IV [24]. Cannabis has a relatively low addiction potential, and dependence appears to be less severe compared to tobacco, alcohol and other substances $[4,7,8,27,28]$. There is a large consensus that both an increased tolerance and a withdrawal syndrome (if existing) are less evident in contrast to other substances [7].

Despite the decrease in global cannabis use, in the past decade, the number of admissions for first-time entrants seeking treatment for substance use disorders with cannabis as principal reason substantially increased in Western and Central Europe [2, 17]. However, the overall number of cannabis clients entering specialized drug treatment stabilized in 2012. In the United States, the number of persons receiving treatment in the past year for cannabis was stable between 2002 and 2012 [16].

To date, plenty of studies have examined potential factors associated with the first onset of cannabis use and abuse. However, there is less research on potential conditions under which cannabis dependence is more likely to emerge $[19,29,30]$. Specifically, this research topic is of considerable public health importance due to potentially adverse effects like absence from work/school, health problems, treatment costs and conflict with the law [31]. Due to increasing rates of individuals requesting treatment for cannabis, there is a greater need for scientifically based knowledge on precursors of dependence. Furthermore, a better understanding of these determinants might help to improve existing interventions and to develop adequate preventions. Although previous studies have examined different potential determinants of cannabis dependence, to our knowledge, a systematic review is lacking.

The primary aim of our review was to systematically summarize evidence for psychosocial factors associated with the development of cannabis dependence and to fill the scientific gap on this topic. The term psychosocial determinant comprises individual issues (like personality traits, mental disorders) and social environments associated with the occurrence of dependence. We define cannabis dependence as a diagnosis in conformity with the ICD-10 and DSM-IV which is characterized by increased 
tolerance, using cannabis in greater amounts or over a longer period of time than intended and difficulties in controlling its use [24-26].

\section{Methods}

Search Strategy

A search on relevant studies regarding predictors of cannabis dependence was conducted on 4 databases: Embase, Medline, PsycINFO and PSYNDEX. The last search was run on May 15, 2014. Our search strategy involved using various combinations of $\mathrm{MeSH}$ terms and keywords. Search terms used in both search strategies were 'cannabis dependence OR marijuana dependence OR cannabis addiction OR marijuana addiction', combined with 'risk factor', 'risk factors', 'factor', 'factors', 'determinant', 'determinants', 'predictor' and 'predictors'. German equivalents were used in the same way.

\section{Study Selection}

Only papers examining psychosocial predictors of cannabis dependence were considered, that is, mental disorders, intrapersonal variables, onset of cannabis use and patterns of consumption, psychotropic reactions and motives of cannabis use and social correlates. First, our search detected a total of 10,568 potentially relevant articles. All searches were limited to papers published in English and German, with humans as subject targets. Moreover, a 14-year restriction on publication years (2000-2014) was applied for all searches. In addition, each study had to meet further inclusion criteria: (a) studies examining dependence upon various substances had to evaluate cannabis dependence separately and (b) specifically, an odds ratio or a $\mathrm{p}$ value to indicate statistical significance of relationships was required.

Potentially eligible papers were selected by screening the titles (first step), abstracts (second step) and the entire article (third step) retrieved through the database searches. A flowchart according to the PRISMA statement [32] contains the exclusion criteria for papers assessed in this review (fig. 1). Both duplicate papers and titles identified as irrelevant were removed, leaving a total of 2,799 papers. Screening abstracts resulted in a selection of 166 articles for full-text review. One hundred and forty of these papers did not meet the above mentioned inclusion criteria, resulting in a final inclusion of 26 relevant articles. Subsequently, information was extracted from each included study on author(s), year of publication and sample size, as well as main results (tables 1 and 2).

\section{Results}

A total of 26 papers were included, containing 15 longitudinal (or panel), 10 prospective or clinical and 1 longitudinal qualitative study. Most of these studies $(n=14)$ focused on adolescents and young adults (age group 1430 years). Overall, a wide range of predictor variables was identified in the studies and can be subdivided into the following 4 categories: (1) comorbid mental disorders and intrapersonal variables, (2) onset of cannabis use and use patterns, (3) reactions to and motives for cannabis use and (4) social correlates of cannabis dependence.

\section{Comorbid Mental Disorders and Intrapersonal Variables}

The prevalence of mental disorders was substantially higher for dependent than non-dependent cannabis users [33]. Furthermore, the majority of studies examining comorbidity focused on substance-related disorders and on affective disorders. It was estimated that comorbid tobacco dependence increases the probability for regular cannabis use and cannabis dependence. In a 10-year cohort study, the rates of development of cannabis dependence among young adults who smoke cigarettes in high doses significantly increased. Former daily smokers in adolescence had a 27 -fold elevation in risk for cannabis dependence compared to nonsmokers [34]. Moreover, cannabis-dependent respondents have also been reported to be at increased risk for current smoking [35]. Longitudinal studies confirmed that persistent cigarette smoking in teenage years correlated with cannabis dependence diagnosis [36, 37]. Moreover, cannabis dependence was strongly related to tobacco dependence [38]. In one study, however, the development of a cannabis dependence syndrome was associated with neither concomitant illegal drug use nor comorbid tobacco dependence [39].

In another study, cannabis use appeared to be a marker of cannabis dependence symptoms only in participants who consumed alcohol frequently or in large amounts [40]. Thus, cannabis in combination with alcohol use, rather than cannabis use alone, may lead to cannabis dependence. In another work, lifetime diagnosis of alcohol dependence has been found to have an impact on lifetime cannabis dependence [38]. However, Nocon et al. [39] did not show consistent relationships between cannabis dependence and alcohol dependence. Surprisingly, a longitudinal study by Coffey et al. [36] found that persistent frequent drinking in adolescence protects against the development of cannabis dependence: there was no increased risk of later dependence for weekly or daily cannabis users with frequent alcohol use. On the contrary, weekly or daily cannabis use co-varied with cannabis dependence in people who rarely drank alcohol [36]. The results of von Sydow et al. [41] confirmed alcohol, specifically its availability at home, as a protective factor against cannabis dependence.

Moreover, baseline use of other illicit drugs was found to play a significant role in the transition to cannabis dependence [41]. Consistent with the finding of von Sydow 
Fig. 1. PRISMA flow diagram of study se-

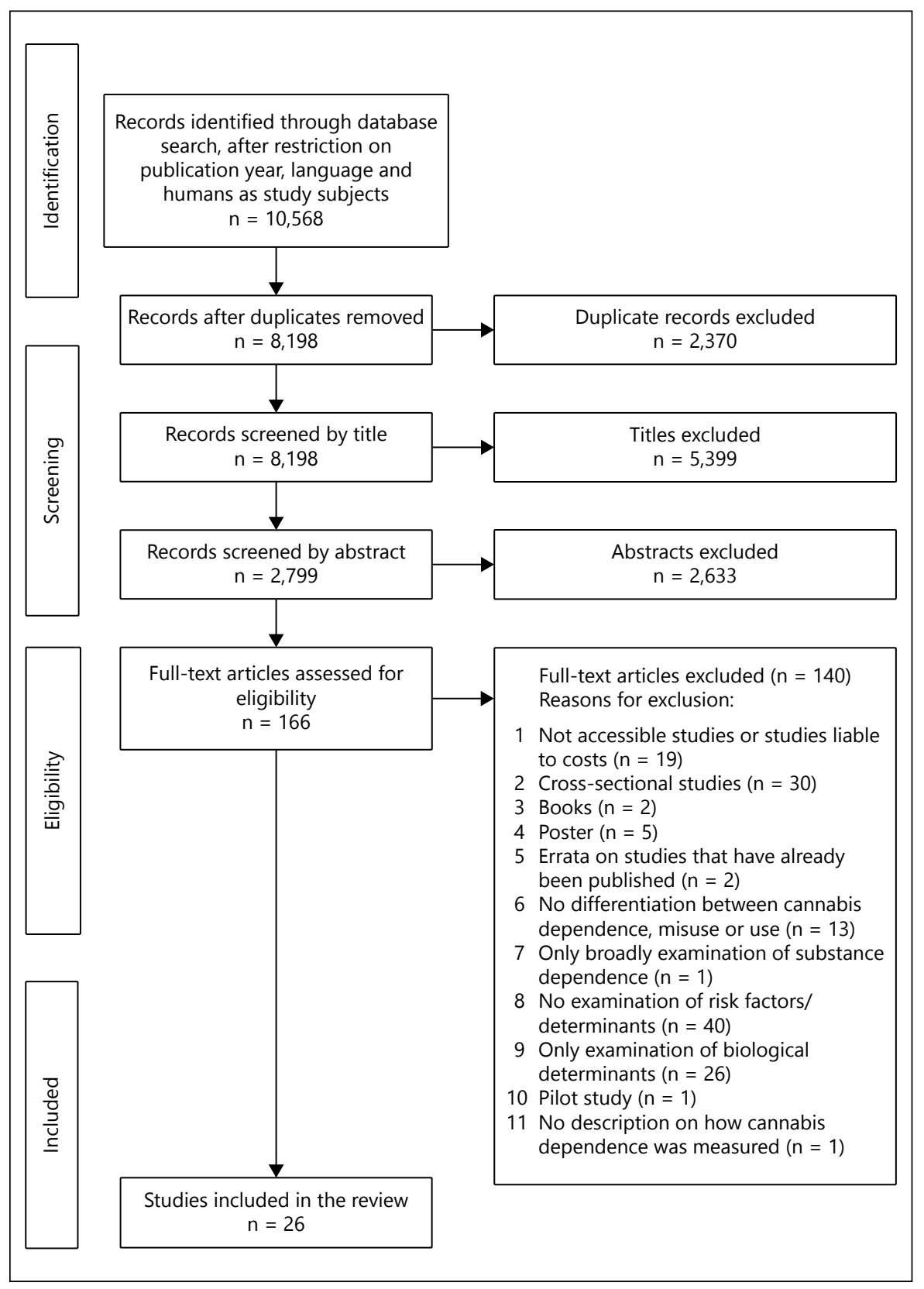

et al. [41], Chen et al. [42] showed that the use of 3 or more drugs prior to starting cannabis marked a threshold of risk for later dependence. In addition, some evidence suggests that a lifetime prevalence of cannabis dependence correlated positively with cocaine abuse [43]. However, cannabis dependence seems not always to be necessarily determined by the use of other illicit substances [39].

Individuals with a non-medical use and overuse of medically prescribed stimulants for attention deficit hyperactivity disorder (ADHD) exhibited significantly higher rates of cannabis dependence compared to those not using stimulants [44]. Among individuals with baseline major depression, Pacek et al. [45] found, in a 3-year longitudinal study, 4.5 times higher probability of developing co-occurring alcohol and cannabis dependence at follow-up than those without baseline depression. Furthermore, respondents who experienced an independent major depression (outside the context of drug use) were significantly more likely to have cannabis dependence than those who had depression onset at the same time or 


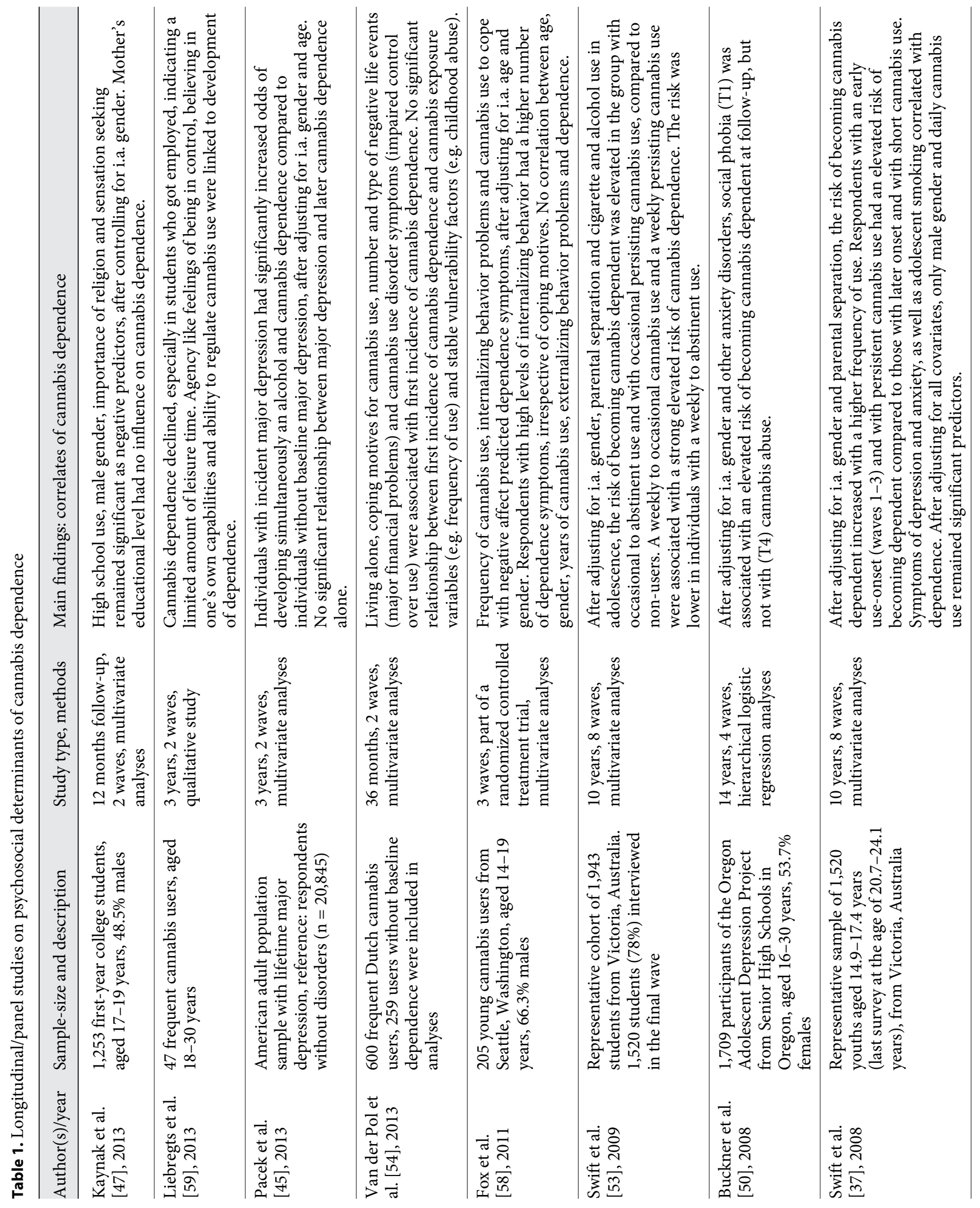




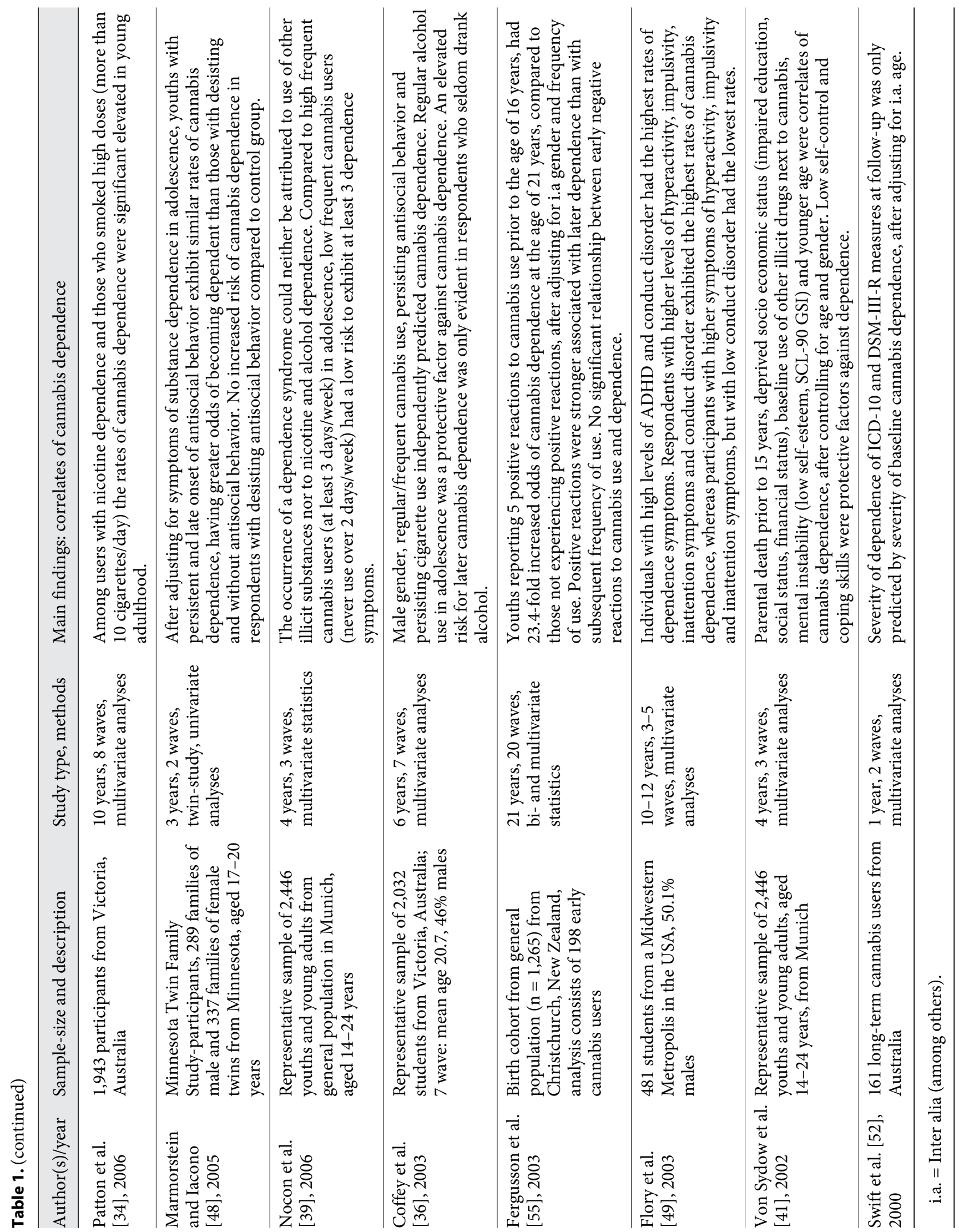




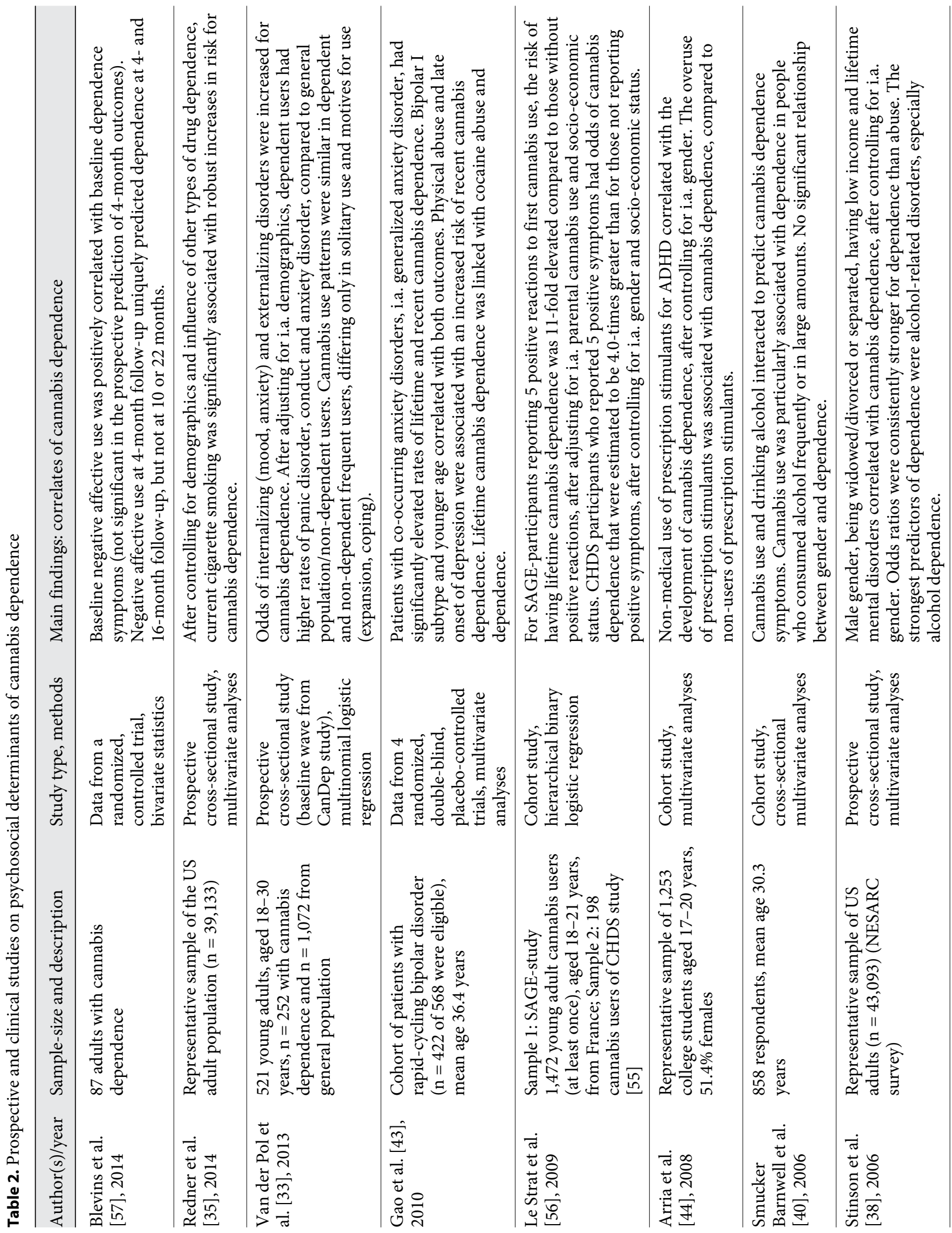




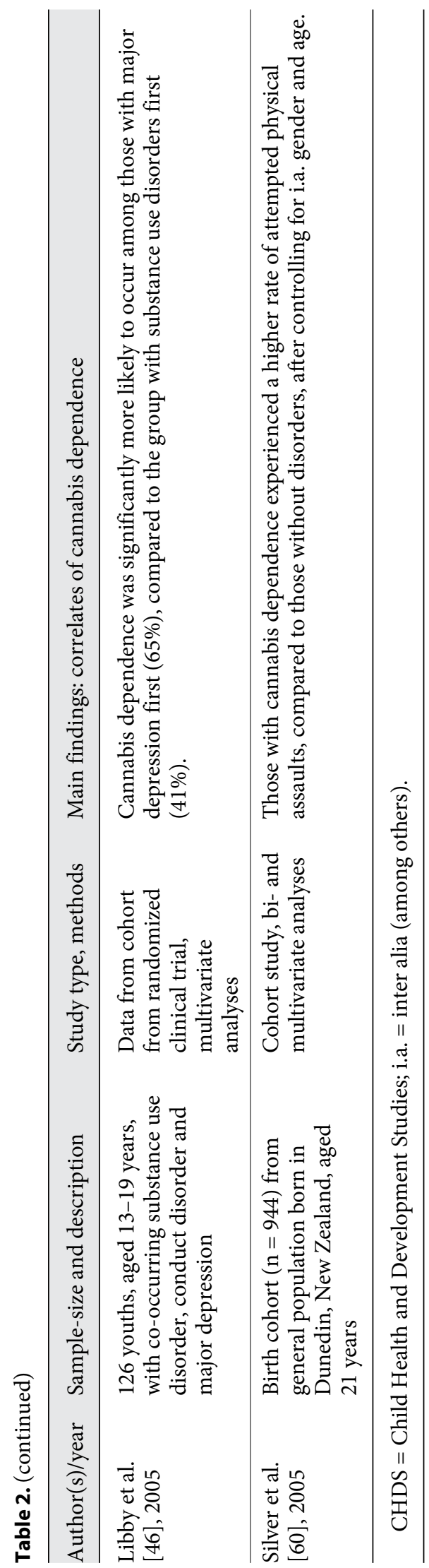

later [46]. However, one paper did not find significant interaction between cannabis dependence and major depression [41]. In other studies, higher rates of anxiety disorders, dysthymia and bipolar disorders have been noted among dependent individuals [33, 38, 43].

The role of panic disorder in development of dependence has been mixed. Indeed, lifetime and past-year history of panic disorder may also exert its influence on the onset of cannabis dependence [33, 38]. However, in the study by Gao et al. [43], history of panic disorder was not a predictor of cannabis dependence. In one study, generalized anxiety independently predicted recent and lifetime cannabis dependence among patients with rapid cycling. One study found significant associations between higher sensation-seeking levels and cannabis dependence [47].

Moreover, the odds of cannabis dependence were significantly greater among persons with antisocial behavior [36]. In a twin study, Marmorstein and Iacono [48] found similar higher rates of cannabis dependence in those of 17-20 years with antisocial behavior expressed before and after 15 years of age (persisting vs. late onset), compared to control group. In contrast, adolescents with desisting antisocial behavior were not at increased risk for dependence. Further findings demonstrated that young adults with a history of a higher number of symptoms of both ADHD and conduct disorder had the highest rates for cannabis dependence symptoms [49]. Similarly, van der Pol et al. [33] showed that dependent cannabis users had greater odds of conduct disorder, compared to nonusers and non-frequent users in the general population. Interestingly, those individuals with higher symptoms of ADHD but lower conduct disorder had the lowest dependence rates [49].

In a longitudinal study, social anxiety disorder was a prominent predictor for the subsequent onset of cannabis dependence, remaining significant after controlling for other anxiety disorders [50]. In addition, cannabis dependence was more likely to occur in persons with higher behavioral inhibition during childhood (fear factor, social factor) and with indicators of mental instability like a higher SCL-90-GSI score and low self-esteem. Surprisingly, low self-control and coping skills had a protective effect [41].

All in all, many studies discussed the relationship between cannabis dependence and comorbid mental disorders or symptoms in the context of the self-medication hypothesis [51]. This model suggests that individuals with mental problems use substances like cannabis to relieve psychological distress and to cope with negative affect leading to regular use and to subsequent dependence. 
In contrast, there is an alternate hypothesis that implies the existence of dependence, causing neurobiological changes that increase the likelihood of comorbid mental disorders [46].

\section{Onset of Cannabis Use and Use Patterns}

In the longitudinal study by Nocon et al. [39], the risk of developing dependence appeared in a dose-dependent manner: adolescents who tended to use cannabis frequently had a greater likelihood of being classified as dependent, compared to less frequent users. Similarly, severity of dependence at baseline was the primary predictor of dependence at follow-up [52].

The findings by Swift et al. [37] indicated that an early onset of cannabis use and persistent cannabis use were markers of increased risk of cannabis dependence. Adolescents with daily and weekly use in adolescence (aged 1417) were more prone to develop dependence later at 24 years of age [37]. However, in the study by Coffey et al. [36] weekly use rather than daily use was more predictive of cannabis dependence. Other findings have shown that respondents with weekly persisting and with a weekly occasional cannabis use had markedly elevated odds for dependence. Compared to this high-risk group, people with less frequent use had a lower risk of developing dependence - even though the risk still remained elevated compared to nonusers [53]. In contrast to the persisting use pattern, a pattern of moderating or decreasing adolescent cannabis use was associated with less risk of dependent use [53]. Hence, among frequent cannabis users, periods of abstinence rather than reduced use may be beneficial to future use patterns. Moreover, younger age at first cannabis use appeared to be of crucial importance to development of dependence [41$43,47]$. The highest probability of becoming dependent was found in users with first use at the age of 14-15 years. From that point on, the estimated risk ratios decreased with age resulting in the lowest risk in onset users at 18-20 years of age [42]. However, in one study, cannabis use patterns and exposure variables like mean age of first onset of use and use frequency appeared to be strikingly similar when comparing dependent with non-dependent users - except for solitary use and coping motives [54]. Accumulating evidence suggests that solitary cannabis use appears as a significant predictor of dependence $[33,54]$.

\section{Psychotropic Reactions to Cannabis Use and Motives for Cannabis Use}

Scientific evidence suggests that a high number of positive reactions to adolescent cannabis use (like getting high, feeling relaxed, laughing a lot) was strongly predic-

Psychosocial Determinants of Cannabis Dependence tive of later dependence at the age of $18-21$ years $[55,56]$. In line with the findings of Fergusson et al. [55] in the longitudinal Christchurch Health and Development Study, Le Strat et al. [56] reported that increasing negative reactions to first cannabis use were not related to later dependence.

In addition, using cannabis to cope with negative affect predicted the number of cannabis dependence symptoms [57-59]. Adolescents with or without low internalizing behavior problems (like anxiety sensitivity or depression symptoms) who use cannabis to cope with negative situations were at a high risk for cannabis dependence. However, there was no interaction between externalizing behavior problems and coping motives in order to predict cannabis dependence [58]. Van der Pol et al. [33] found that cannabis dependence was associated with robust increases in risk for both internalizing (mood, anxiety) and externalizing disorders (delinquency, aggression). Furthermore, coping motives and positive affective use correlated positively with dependence $[33,54,57]$.

\section{Social Correlates of Cannabis Dependence}

Adolescents dependent on cannabis significantly differed from their non-dependent counterparts in family structure, arising significantly more often from singleparent or blended families [56]. In another work, not living with parents has been found to have an impact on cannabis dependence $[41,56]$. Among youths experiencing parental separation at baseline, Coffey et al. [36] found a 2-fold elevated risk of dependence.

Parental death before age 15 years was considered a marker for the transition to dependence among former users without disorder. Availability of drugs and influence of the peer group predicted only cannabis use, not dependence. Moreover, none of the study participants dependent on cannabis had a very good relationship with their fathers at baseline [41].

In addition, adolescents whose parents had a history of alcohol dependence were more apt to develop cannabis dependence themselves, especially when socioeconomic disadvantage occurred. Further predictors associated with cannabis dependence were an impaired educational achievement, living alone, being widowed, separated or divorced and a parent with higher cannabis use $[38,54$, 56]. Specifically, a qualitative study by Liebregts et al. [59] revealed that a limited amount of leisure time and employment were protective factors against cannabis dependence. Furthermore, being a victim of attempted physical assault might have contributed to young adults' risk of cannabis dependence [60]. A history of physical abuse has 
been documented to occur at a higher rate for current cannabis dependence [43].

Accumulating evidence suggests that cannabis dependence tends to occur more often among males than among females [36, 38, 47]. Other studies, however, did not show significant interaction with gender [41, 42]. A deprived socioeconomic situation (low education, low social status, bad financial situation) has been reported to be an important precursor of the development of cannabis dependence among youths and young adults [41]. This is in line with other findings, where an excess risk of cannabis dependence was found for recent-onset users with a low family income and major financial problems $[38,42,54]$. Furthermore, no significant association between ethnicity and cannabis dependence was found after controlling for covariates [42, 47].

\section{Discussion}

All in all, the present knowledge gained on predictors of cannabis dependence is rather ambiguous. This underlines the complexity of interactions between various factors and the transition to dependence. Evidence suggests that development of the dependence syndrome appears to be a product of several biographic and accompanying processes.

In summary, the most consistent predictive factors of cannabis dependence were an early onset of cannabis use (at the age of 11-15 years), frequent use, positive psychotropic effect of cannabis, use independent from social contexts and prior drug involvement. Furthermore, comorbid mental disorders like affective disorders, anxiety disorders and alcohol-related disorders also seem to predict first incidence of dependence. In addition, stressful and critical life events like physical abuse, parental separation, early parental death or an impaired socioeconomic status seem to play a crucial role in transition to cannabis dependence. Altogether, it emerges that factors predicting the onset of cannabis use and abuse differ from those influencing progression to dependence $[38,41,50$, 63]. Positive attitudes toward future drug use, availability of drugs and peer drug use were associated with incident cannabis use but not with dependence. In contrast, male gender and drug disorder at baseline showed the strongest association with cannabis abuse. On the other hand, cannabis dependence has been linked to greater levels of impairment (financial, familial and psychopathological) and correlated with using other illicit drugs. Other studies showed social anxiety disorders and panic attacks being solely associated with cannabis dependence, not with abuse [50,63]. However, only few studies assessed predictors of both cannabis abuse and dependence.

\section{Conclusion and Implications}

Numerous factors were shown to have an impact on transition to cannabis dependence. In particular, a wide range of mental disorders has been linked to an elevated risk of developing cannabis dependence. One can infer from some studies that cannabis use alone will lead directly to dependence [52]. Nevertheless, low rates of dependence disorder among users seem to contradict this hypothesis [61]. Nocon et al. [39] found that (frequent) use alone does not have to be necessarily linked with cannabis dependence; even regular cannabis use of 2 days per week was associated with a relatively low risk of becoming dependent [39]. It appears that adverse circumstances like traumatic experiences or comorbid mental disorders play a crucial role in this process - even though it cannot be proven beyond a doubt. Nevertheless, in interpreting the results, it should be taken into account that the causality of variables remains unclear. On the one hand, results may describe a causal association, as in the case of mental disorders contributing to an increased risk of cannabis dependence. Here, cannabis users may consume it as an attempt to self-medicate. It is also possible that use may enhance or cause symptoms of comorbid disorders. On the other hand, the examined variables can be indirectly associated with dependence, which again is related to further factors. There might also be a non-causal relationship between these variables - for instance, when specific genetic predispositions or social and environmental factors are shared.

An appropriate intervention should depend on the stage of substance use in which the intervention occurs. Prevention of cannabis dependence should focus on individuals with comorbid disorders who experienced parental death, live under impaired socioeconomic conditions, have already used other illicit drugs and report positive psychotropic reactions to cannabis use [41].

Furthermore, it is important to examine coping motives for use because motives for dependent use appeared to differ from those for occasional use. This is in line with evidence from some studies; the notion was put forward that persons dependent on cannabis might differ from infrequent and non-dependent users in having other motives like wishing to reduce negative affect and tension, to cope with stressors or to manage symptoms. Believing that it may help, they would tend to use regularly, which may result in dependence $[62,63]$. Finally, evidence sug- 
gests that the younger the age at first cannabis use, the more vulnerable adolescents are to dependence. Therefore, a delay of cannabis use-onset to young adulthood may be a possible intervention strategy.

\section{Possible Limitations}

These results should be regarded in the context of possible limitations of this review; in order to strengthen the consistency of the review we aimed at creating a clear and well-defined basis, so we considered only articles describing cannabis dependence and its synonyms as variables. Papers with terms like abuse, excessive use and frequent use were excluded due to inconsistent and vague definitions. Furthermore, we did not consider biological determinants for review, so the determinants in their entirety could not be ascertained. We chose a 14-year restriction on publication years because there has been a recent rapid growth in research on cannabis dependence. Particularly in the last decades, increasing attention was paid to cannabis and the accompanying processes. Therefore, we decided to use an economic approach examining the recent literature in order to gain the most current scientific insights into this topic. Moreover, a different procedure in giving a diagnosis of cannabis dependence and in the number of considered criteria for dependence hindered comparability between the studies. Accordingly, there remains considerable uncertainty about the validity and the chance to make firm conclusions. Nevertheless, the included studies address an important question reporting on dependence upon cannabis. Simultaneously, the limited validity of some studies makes clear that a more consistent and precise use of definitions and dependence criteria across studies would be beneficial. We did not consider mere cross-sectional studies in order to ensure a higher degree of internal validity. However, when taking this type of study into account, one could get a broader range of correlates associated with cannabis dependence. These further factors identified in cross-sectional studies may affect the likelihood of developing dependence, for instance, trait anxiety, physical anhedonia, borderline personality disorder, psychological distress, avoidant coping, suicidal ideation, sexual abuse and combat exposure adjusted for combat-related post-traumatic stress disorder accounted for a significantly elevated risk of cannabis dependence $[29,60,64-69]$. Furthermore, use patterns like blunt use or water pipe use were predictive of cannabis dependence in cross-sectional studies [30, 70, 71].

Taken together, only one study controlled for multiple substance use [55]. However, when simultaneous use of at least 2 substances has been reported, it appears to be (almost) impossible to consider the effect of a single substance separately from one another. For example, using alcohol in addition to cannabis can cause its own problems, which might be wrongly attributed to cannabis use [61]. Furthermore, it is difficult to examine the quantity of cannabis use, due to huge variation in potency, use patterns, duration of use and so on. Although many studies controlled for various factors in order to eliminate confounding factors from the relationship, some did not consider them in regression analyses [34, 40, 43, 49, 53]. In addition, few papers examined variables other than gender and age, which might provide plausible alternative explanation about the observed associations. The twin study [48] was well suited as a control technique because it provided control over predisposing factors resulting from similar environment and genes. In many studies, either no control group existed or individuals dependent on cannabis were compared with different groups (other cannabis users, non-users), which impeded comparability. The majority of studies recruited a large number of participants $(n>1,000)$, only in one study, a too small sample size limited statistical significance [46]. It is, however, problematic that studies consisting of general-population samples identified only a small number of cannabis-dependent users [41]. Some studies selected participants by respondent driven sampling or other forms of convenience sampling (e.g., users were recruited in coffee shops). Indeed, they make useful contributions because they directly addressed the target group and recruited a larger sample. However, they are prone to biases and evidence cannot be generalized to normal population as they consist of non-representative samples.

Some of the included studies made cannabis dependence diagnoses that were partly not in line with ICD-10 or DSM-IV. Specifically, the diagnosis was made when 2 instead of the minimum of 3 required criteria were fulfilled. Generally, the given diagnoses appeared somewhat arbitrary and the comparability between studies was hindered because of different measures (e.g., classification of cannabis users, often lacking a specification of use methods) and blurry descriptions.

Furthermore, it should be also taken into account that non-users may differ from users in variables other than non-use. With regard to frequency of use, evidence suggests that daily use is not necessarily associated with dependence. Conversely, non-daily use does not automatically imply a lack of dependence [72, 73]. In addition, evidence from most studies only disclosed correlates as- 
sociated with persistence of cannabis use once dependence has developed. However, the process after transition to dependence itself may be a causal determinant of the subsequent cannabis use [42].

\section{Future Prospects and Ways for Future Research}

From the revised criteria of DSM-5, it follows that the DSM-IV categories for substance use and abuse were subsumed under substance-related addictive disorders [25]. On one hand, operating on a continuum makes it needless for clinicians to discriminate between both diagnoses as cannabis abuse and dependence not always appearing as cut and dried entities in practice. Moreover, the new conceptualization allows specifying severity of substance use disorder depending on the number of symptoms. On the other hand, the single category substance use disorders may lead to a higher number of diagnoses since merely 2 criteria have to be fulfilled. Consequently, estimates of its prevalence would increase significantly by augmenting slightly higher rates of cannabis abuse with comparatively lower dependence rates. On the contrary, an extension of the former categories might offer the chance to explore dependence and abuse in a comprehensive and global way. However, as there is no need to operate with distinct diagnoses, boundaries of the dependence concept somehow become blurred lacking clear and well-defined criteria. This, in turn, might increase the likelihood of cannabis users receiving a diagnosis. Consequently, the assessment of the course of dependence rates and the comparability between actual and previous rates would be hindered or even turn out to be impracticable. In addition, as substance abuse usually precedes dependence and is likely to be predicted by different factors it would be beneficial to discriminate between both terms, particularly with regard to appropriate interventions.

For future work, it will be important to recruit larger representative samples through epidemiological methods using valid and comparable instruments in order to make findings generalizable. While doing this systematic review, it was striking that some studies used terms like problematic use, abuse and dependence while lacking an exact operationalization or using the term dependence even if the required number of symptoms was not fulfilled. With regard to frequency of use, one should achieve agreement on the number of days cannabis was used or on the number of joints used in a given period of time. This can be completed by gathering further information on use method, quantity and quality (type, potency). The knowledge of context, motives and subjective effects could lead to a broader understanding of the disorder in order to develop appropriate prevention and intervention.

\section{Disclosure Statement}

The authors report no conflicts of interest.

\section{References}

1 Budney AJ, Lile JA: Moving beyond the cannabis controversy into the world of the cannabinoids. Int Rev Psychiatry 2009;21:91-95.

2 European Monitoring Centre for Drugs and Drug Addiction (EMCDDA): European drug report 2014: trends and developments. 2014. http://www.emcdda.europa.eu/publications/ edr/trends-developments/2014.

3 Reuter PH: Marijuana legalization: what can be learned from other countries. Santa Monica, RAND: Drug Policy Research Center, 2010. http://www.rand.org/content/dam/rand/ pubs/working_papers/2010/RAND_WR771. pdf.

4 Van den Brink W: Forum: decriminalization of cannabis. Curr Opin Psychiatry 2008;21: 122-126.

5 Bonnet U, Scherbaum N: Cannabisbezogene Störungen. Teil II: Psychiatrische und somatische Folgestörungen und Komorbiditäten [Cannabis use disorders. Part II: psychiatric and somatic sequelae and comorbidities]. Fortschr Neurol Psychiatr 2010;78:360-367; quiz 368.
6 Soellner R, Gabriel U: Typisch »Kiffer«? Stereotype und Personenwahrnehmung [Typical pothead? Stereotypes and person perception]. Sucht 2008;54:32-37.

7 Jager G: Cannabis; in Verster J, Brady K, Galanter M, Conrod P (eds): Drug Abuse and Addiction in Medical Illness: Causes, Consequences and Treatment. New York, Springer, 2012, pp 151-162.

8 Murray RM, Morrison PD, Henquet C, Di Forti M: Cannabis, the mind and society: the hash realities. Nat Rev Neurosci 2007;8:885895.

9 Bostwick JM: Blurred boundaries: the therapeutics and politics of medical marijuana. Mayo Clin Proc 2012;87:172-186.

10 Grotenhermen F, Müller-Vahl K: The therapeutic potential of cannabis and cannabinoids. Dtsch Arztebl Int 2012;109:495-501.

11 Pacher P, Bátkai S, Kunos G: The endocannabinoid system as an emerging target of pharmacotherapy. Pharmacol Rev 2006;58: 389-462.
12 Congressional Research Service: State legalization of recreational marijuana: selected legal issues. 2014. http://fas.org/sgp/crs/misc/ R43034.pdf.

13 Hawken A, Caulkins J, Kilmer B, Kleiman M Quasi-legal cannabis in Colorado and Washington: local and national implications. Addiction 2013;108:837-838.

14 Sevigny EL, Pacula RL, Heaton P: The effects of medical marijuana laws on potency. Int $J$ Drug Policy 2014;25:308-319.

15 Bonnet U, Scherbaum N: Cannabisbezogene Störungen. Teil I: Pharmakologie, Epidemiologie und Therapieverfahren [Cannabis use disorders. Part I: pharmacology, epidemiology and therapy]. Fortschr Neurol Psychiatr 2010;78:297-305.

16 National Survey on Drug Use and Health (NSDUH): Results from the 2012 National Survey on Drug Use and Health: Summary of National Findings. 2012. http://www.samhsa.gov/ data/NSDUH/2012SummNatFindDetTables/ NationalFindings/NSDUHresults2012.pdf. 
17 United Nations Office on Drugs and Crime (UNODC): World drug report 2014. https:// www.unodc.org/documents/wdr2014/ World_Drug_Report_2014_web.pdf.

18 Kraus L, Pabst A, Piontek D, Müller S: Trends des Substanzkonsums und substanzbezogener Störungen. Ergebnisse des Epidemiologischen Suchtsurveys 1995-2009 [Trends of substance use and substance use disorders. Results of the 2009 epidemiological survey of substance abuse 1995-2009]. Sucht 2010;56:337-347.

19 Liebregts N, Benschop A, van Der Pol P, van Laar M, de Graaf R, van den Brink W, Korf DJ: Cannabis dependence and peer selection in social networks of frequent users. Contemporary Drug Problems 2011;38:93-120.

20 Noack R, Höfler M, Lueken U: Cannabis use patterns and their association with DSM-IV cannabis dependence and gender. Eur Addict Res 2011;17:321-328.

21 Pabst A, Kraus L, Gomes de Matos E, Piontek D: Substanzkonsum und substanzbezogene Störungen in Deutschland im Jahr 2012 [Substance use and substance use disorders in Germany in 2012]. Sucht 2013;59:321-331.

22 Lopez-Quintero C, Pérez de los Cobos J, Hasin DS, Okuda M, Wang S, Grant BF, Blanco C: Probability and predictors of transition from first use to dependence on nicotine, alcohol, cannabis, and cocaine: results of the National Epidemiologic Survey on Alcohol and Related Conditions (NESARC). Drug Alcohol Depend 2010;115:120-130.

23 Murphy A, Taylor E, Elliott R: The detrimental effects of emotional process dysregulation on decision-making in substance dependence. Front Integr Neurosci 2012;6:101.

24 American Psychiatric Association (APA): Diagnostic and Statistical Manual of Mental Disorders, ed 4. Washington, American Psychiatric Association, 2000.

25 American Psychiatric Association (APA): Substance-related and addictive disorders. American Psychiatric Association, 2013. http://www.dsm5.org/Documents/ Substance\%20Use\%20Disorder\%20Fact\%20 Sheet.pdf.

26 World Health Organization (WHO): International Statistical Classification of Diseases and Related Health Problems, ed 2. Geneva, WHO, 2004.

27 Nutt DJ, King LA, Phillips LD; Independent Scientific Committee on Drugs: Drug harms in the UK: a multicriteria decision analysis. Lancet 2010;376:1558-1565.

28 Van Amsterdam J, Opperhuizen A, Koeter M, Van den Brink W: Ranking the harm of alcohol, tobacco and illicit drugs for the individual and the population. Eur Addict Res 2010; 16:202-207.

29 Cascone P, Zimmermann G, Auckenthaler B, Robert-Tissot C: Cannabis dependence in Swiss adolescents: exploration of the role of anxiety, coping styles, and psychosocial difficulties. Swiss J Psychol 2011;70:129-139.

30 Timberlake DS: A comparison of drug use and dependence between blunt smokers and other cannabis users. Subst Use Misuse 2009; 44:401-415.

31 Taylor DR, Fergusson DM, Milne BJ, Horwood LJ, Moffitt TE, Sears MR, Poulton R: A longitudinal study of the effects of tobacco and cannabis exposure on lung function in young adults. Addiction 2002;97:10551061.

32 Moher D, Liberati A, Tetzlaff J, Altman DG; PRISMA Group: Preferred reporting items for systematic reviews and meta-analyses: the PRISMA statement. Ann Intern Med 2009; 151:264-269.

33 van der Pol P, Liebregts N, De Graaf R, Ten Have M, Korf DJ, van den Brink W, van Laar M: Mental health differences between frequent cannabis users with and without dependence and the general population. Addiction 2013;108:1459-1469.

34 Patton GC, Coffey C, Carlin JB, Sawyer SM, Wakefield M: Teen smokers reach their mid twenties. J Adolesc Health 2006;39:214-220.

35 Redner R, White TJ, Harder VS, Higgins ST: Vulnerability to smokeless tobacco use among those dependent on alcohol or illicit drugs. Nicotine Tob Res 2014;16:216-223.

36 Coffey C, Carlin JB, Lynskey M, Li N, Patton GC: Adolescent precursors of cannabis dependence: findings from the Victorian Adolescent Health Cohort Study. Br J Psychiatry 2003; 182:330-336.

37 Swift W, Coffey C, Carlin JB, Degenhardt L, Patton GC: Adolescent cannabis users at 24 years: trajectories to regular weekly use and dependence in young adulthood. Addiction 2008; 103:1361-1370.

38 Stinson FS, Ruan WJ, Pickering R, Grant BF: Cannabis use disorders in the USA: prevalence, correlates and co-morbidity. Psychol Med 2006;36:1447-1460.

39 Nocon A, Wittchen HU, Pfister H, Zimmermann P, Lieb R: Dependence symptoms in young cannabis users? A prospective epidemiological study. J Psychiatr Res 2006;40: 394-403.

40 Smucker Barnwell SV, Earleywine M, Gordis EB: Confirming alcohol-moderated links between cannabis use and dependence in a national sample. Addict Behav 2006;31:16951699.

41 Von Sydow K, Lieb R, Pfister H, Höfler M, Wittchen HU: What predicts incident use of cannabis and progression to abuse and dependence? A 4-year prospective examination of risk factors in a community sample of adolescents and young adults. Drug Alcohol Depend 2002;68:49-64.

42 Chen CY, O'Brien MS, Anthony JC: Who becomes cannabis dependent soon after onset of use? Epidemiological evidence from the United States: 2000-2001. Drug Alcohol Depend 2005;79:11-22.

43 Gao K, Chan PK, Verduin ML, Kemp DE, Tolliver BK, Ganocy SJ, Bilali S, Brady KT, Findling RL, Calabrese JR: Independent predictors for lifetime and recent substance use disorders in patients with rapid-cycling bipo- lar disorder: focus on anxiety disorders. Am J Addict 2010;19:440-449.

44 Arria AM, Caldeira KM, O'Grady KE, Vincent KB, Johnson EP, Wish ED: Nonmedical use of prescription stimulants among college students: associations with attention-deficithyperactivity disorder and polydrug use. Pharmacotherapy 2008;28:156-169.

45 Pacek LR, Martins SS, Crum RM: The bidirectional relationships between alcohol, cannabis, co-occurring alcohol and cannabis use disorders with major depressive disorder: results from a national sample. J Affect Disord 2013;148:188-195.

46 Libby AM, Orton HD, Stover SK, Riggs PD: What came first, major depression or substance use disorder? Clinical characteristics and substance use comparing teens in a treatment cohort. Addict Behav 2005;30:16491662.

47 Kaynak O, Meyers K, Caldeira KM, Vincent KB, Winters KC, Arria AM: Relationships among parental monitoring and sensation seeking on the development of substance use disorder among college students. Addict Behav 2013;38:1457-1463.

48 Marmorstein NR, Iacono WG: Longitudinal follow-up of adolescents with late-onset antisocial behavior: a pathological yet overlooked group. J Am Acad Child Adolesc Psychiatry 2005;44:1284-1291.

49 Flory K, Milich R, Lynam DR, Leukefeld C, Clayton R: Relation between childhood disruptive behavior disorders and substance use and dependence symptoms in young adulthood: individuals with symptoms of attention-deficit/hyperactivity disorder and conduct disorder are uniquely at risk. Psychol Addict Behav 2003;17:151-158.

50 Buckner JD, Schmidt NB, Lang AR, Small JW, Schlauch RC, Lewinsohn PM: Specificity of social anxiety disorder as a risk factor for alcohol and cannabis dependence. J Psychiatr Res 2008;42:230-239.

51 Khantzian EJ: The self-medication hypothesis of addictive disorders: focus on heroin and cocaine dependence. Am J Psychiatry 1985; 142:1259-1264.

52 Swift W, Hall W, Copeland J: One year follow-up of cannabis dependence among longterm users in Sydney, Australia. Drug Alcohol Depend 2000;59:309-318.

53 Swift W, Coffey C, Carlin JB, Degenhardt L, Calabria B, Patton GC: Are adolescents who moderate their cannabis use at lower risk of later regular and dependent cannabis use? Addiction 2009; 104:806-814.

54 Van der Pol P, Liebregts N, De Graaf R, Korf DJ, Van den Brink W, Van Laar M: Predicting the transition from frequent cannabis use to cannabis dependence: a three-year prospective study. Drug Alcohol Depend 2013;133: 352-359.

55 Fergusson DM, Horwood LJ, Lynskey MT, Madden PA: Early reactions to cannabis predict later dependence. Arch Gen Psychiatry 2003;60:1033-1039. 
56 Le Strat Y, Ramoz N, Horwood J, Falissard B, Hassler C, Romo L, Choquet M, Fergusson D, Gorwood P: First positive reactions to cannabis constitute a priority risk factor for cannabis dependence. Addiction 2009;104:17101717.

57 Blevins CE, Stephens RS, Walker DD, Roffman RA: Situational determinants of use and treatment outcomes in marijuana dependent adults. Addict Behav 2014;39:546-552.

58 Fox CL, Towe SL, Stephens RS, Walker DD, Roffman RA: Motives for cannabis use in high-risk adolescent users. Psychol Addict Behav 2011;25:492-500.

59 Liebregts N, van Der Pol P, van Laar M, de Graaf R, van den Brink W, Korf DJ: The role of study and work in cannabis use and dependence trajectories among young adult frequent cannabis users. Front Psychiatry 2013; 4:85.

60 Silver E, Arseneault L, Langley J, Caspi A, Moffitt TE: Mental disorder and violent victimization in a total birth cohort. Am J Public Health 2005;95:2015-2021.

61 Smucker Barnwell S, Earleywine M, Gordis EB: Alcohol consumption moderates the link between cannabis use and cannabis dependence in an internet survey. Psychol Addict Behav 2005;19:212-216.

62 Johnson K, Mullin JL, Marshall EC, BonnMiller MO, Zvolensky M: Exploring the me- diational role of coping motives for marijuana use in terms of the relation between anxiety sensitivity and marijuana dependence. Am J Addict 2010;19:277-282.

63 Zvolensky MJ, Bernstein A, Sachs-Ericsson N, Schmidt NB, Buckner JD, Bonn-Miller MO: Lifetime associations between cannabis, use, abuse, and dependence and panic attacks in a representative sample. J Psychiatr Res 2006; $40: 477-486$

64 Chabrol H, Ducongé E, Casas C, Roura C, Carey KB: Relations between cannabis use and dependence, motives for cannabis use and anxious, depressive and borderline symptomatology. Addict Behav 2005;30:829-840.

65 Clark CB, Perkins A, McCullumsmith CB, Islam MA, Hanover EE, Cropsey KL: Characteristics of victims of sexual abuse by gender and race in a community corrections population. J Interpers Violence 2012;27:1844-1861.

66 Dorard G, Berthoz S, Phan O, Corcos M, Bungener C: Affect dysregulation in cannabis abusers: a study in adolescents and young adults. Eur Child Adolesc Psychiatry 2008; 17: 274-282.

67 Lynskey MT, Heath AC, Nelson EC, Bucholz KK, Madden PA, Slutske WS, Statham DJ, Martin NG: Genetic and environmental contributions to cannabis dependence in a national young adult twin sample. Psychol Med 2002;32:195-207.
68 Lynskey MT, Glowinski AL, Todorov AA Bucholz KK, Madden PA, Statham DJ, Martin NG, Heath AC: Major depressive disorder, suicidal ideation, and suicide attempt in twins discordant for cannabis dependence and early-onset cannabis use. Arch Gen Psychiatry 2004;61:1026-1032.

69 Mathews RR, Hall WD, Gartner CE: Depression and psychological distress in tobacco smokers and people with cannabis dependence in the National Survey of Mental Health and Wellbeing. Med J Aust 2011;195:S12-S15.

70 Noack R, Höfler M, Gründler R, Becker A, Schulz R, Paul K: Konsummuster als Kovariablen von DSM-IV Cannabisabhängigkeit [Relationships between cannabis use patterns and DSM-IV cannabis dependence]. Sucht 2009;55:137-147.

71 Ream GL, Benoit E, Johnson BD, Dunlap E: Smoking tobacco along with marijuana increases symptoms of cannabis dependence. Drug Alcohol Depend 2008;95:199-208.

72 Chen CY, Anthony JC: Possible age-associated bias in reporting of clinical features of drug dependence: epidemiological evidence on adolescent-onset marijuana use. Addiction 2003;98:71-82.

73 Swift W, Hall W, Didcott P, Reilly D: Patterns and correlates of cannabis dependence among long-term users in an Australian rural area. Addiction 1998;93:1149-1160. 